\title{
Stellungnahme zum Histoscanning
}

A Iter hat auch seine Vorzüge: Man erkennt die Uniformität sich wiederholender Vorgänge und darf sich wundern. Die Sau, die jeweils durchs urologische Dorf getrieben wurde, hat nur unterschiedliche Namen: HIFU, Kryotherapie, TUMT, Grüner Laser. Allen Verfahren gemeinsam ist das Zielorgan (Prostata) und eine gewisse Energie, die bei der Anwendung appliziert wird und bei der Verbreitung der Methode erforderlich ist. Meist tritt die Sau ihren Weg einigermaßen ernährt, hoffnungsvoll und vielversprechend an. Ist auf den ersten Etappen das Futter noch gut, so wird es später karg - so karg, dass Zeichen der Dystrophie erkennbar werden und der Tod droht. So geht es medizinischen apparativen beziehungsweise instrumentellen Verfahren (zu denen auch TRUS-ANNA gehört), die ohne ausreichende Vorgabe wissenschaftlicher Daten (wie bei Arzneimitteln gefordert), auf die Beine kommen sollen. Werden nicht von der ersten Stunde nach Einführung auf solide Weise (Randomisierung ist kein Zwang) Daten generiert, gibt es zwei Gewinner: die Industrie und die ärztlichen Pioniere, und zwei Verlierer, die Patienten und die Krankenkassen.

\section{Eingehende Prüfung steht aus}

Jetzt haben wir es also mit Histoscanning zu tun, einem Verfahren, das Stadium und Lokalisation des Primärtumors präzise abklären soll. Die Autoren beschreiben kurz die Technik. Wie kann aber diese Technik an den Mann gebracht werden? Wie bei den eingangs genannten Verfahren wird auch diese Methode beworben, ohne dass Daten eingehender Prüfungen vorliegen. Neu ist die Tatsache, dass der kollektive Geist einiger Kollegen so weit reichen soll, gemeinsam an 15 sogenannten Diagnosezentren, das sind die Standorte der Ultraschall-Geräte sowie des Histoscannings, in zwei Jahren mit 15 bis 20 Urologen pro Jahr 600 Patienten zu untersuchen. Das ist die erreichbare Zahl von jährlich 30 bis 40 Probanden pro kooperierendem Kollegen. Um die in dem Beitrag gestellten vier Fragen zu beantworten, wurden Hypothesen generiert, die zwar plausibel sind, die sich jedoch nicht auf Studien stützen. Wenn die Fir-

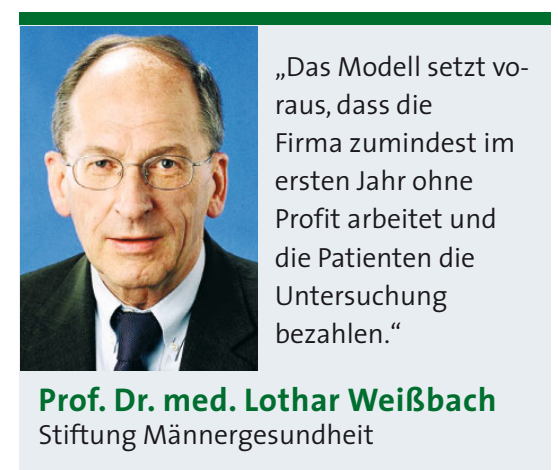

ma Advanced Medical Diagnostics in die Vorleistung geht, indem sie die Gerätschaften kostenfrei verfügbar macht und wenn die beteiligten Kollegen die Forderung nach einheitlichem Vorgehen bei der Untersuchung, bei Dokumentation der initialen und der Folgedaten und bei der Auswertung erfüllen, dann kann der Wert der Methode wie in einer Phase-II-Studie geprüft werden. Die Kostenfrage ist offenbar so geregelt, dass den Krankenkassen keine Zusatzkosten entstehen und die in einer Teilberufsausübungsgemeinschaft arbeitenden Kollegen eine Aufwandsvergütung erhalten. Das setzt aber voraus, dass die Firma zumindest im ersten Jahr ohne Profit arbeitet und die Patienten die Untersuchung bezahlen.

\section{Chancen für das Modell}

Ein solches Modell führt leicht zur Dystrophie, weil

- kein Antrag bei den einschlägigen bekannten Förderinstitutionen eingereicht wurde (obwohl dort Urologen an den Entscheidungen beteiligt sind) und

- Patienten eine wissenschaftliche Untersuchung finanzieren.

Trotzdem kann es erfolgreich sein, weil -Urologen die Gemeinsamkeit suchen, um durch Datengenierung eine $\mathrm{Me}$ thode wissenschaftlich zu prüfen.

Allzu gern lässt man sich nach vielen Jahren Urologie noch angenehm überraschen. Dann müssten die Initiatoren eine wissenschaftliche Begleitung durch eine Studienleitung anstreben, ein Protokoll vorlegen, einen wissenschaftlichen Beirat installieren und die Ethikkommission informieren. 